\title{
MicroRNA-146a inhibits cell migration and invasion by targeting RhoA in breast cancer
}

\author{
QIN LIU ${ }^{1}$, WEI WANG ${ }^{1}$, XIONGFA YANG $^{2}$, DONGXIAO ZHAO $^{1}$, FANGQIONG LI $^{1}$ and HAI WANG ${ }^{1}$ \\ ${ }^{1}$ Department of Clinical Laboratory, Tongde Hospital of Zhejiang Province; ${ }^{2}$ Key Laboratory of Organosilicon Chemistry and \\ Material Technology of the Chinese Education Ministry, Hangzhou Normal University, Hangzhou, Zhejiang 310012, P.R. China
}

Received December 31, 2015; Accepted February 11, 2016

DOI: $10.3892 /$ or.2016.4788

\begin{abstract}
MicroRNAs (miRNAs) function as genetic modulators that regulate gene expression and are involved in a wide range of biological roles, including tumor cell migration and invasion. In the present study, we demonstrated that the migration and invasion activity in MDA-MB-231 breast cancer cells could be directly influenced by altering miR-146a expression. The expression of RhoA and miR-146a in the breast cancer cells showed an inverse correlation. Upregulation of miR-146a in the MDA-MB-231 breast cancer cells by transfection of miR-146a mimics resulted in decreased RhoA protein levels. Conversely, downregulation of miR-146a by transfection of miR-146a inhibitor resulted in increased RhoA protein levels. To confirm the fact that RhoA is a potential target of miR146a, luciferase reporter containing the RhoA 3' untranslated region ( $3^{\prime}$ UTR) was constructed. The results demonstrated that the luciferase reporter activity was reduced after overexpression of miR-146a. Moreover, the luciferase reporter which was constructed with the RhoA 3'UTR mutant did not show significantly altered luciferase reporter activity. Furthermore, after treatment with the RhoA inhibitor exoenzyme C3 transferase protein, the migratory capacity of the MDA-MB-231 cells was not significantly altered even though the amount of miR-146a was changed. Our results indicate that miR-146a functions as a tumor suppressor in breast cancer cells. Downregulation of the expression of miR-146a increased the migration of MDA-MB231 cells, due to the upregulation of RhoA expression.
\end{abstract}

\section{Introduction}

Breast cancer is the most common cancer among women, and its mortality rate ranks second only after lung cancer (1). Metastasis is the main reason for the mortality of breast cancer

Correspondence to: Qin Liu, Department of Clinical Laboratory, Tongde Hospital of Zhejiang Province, 234 Gucui Road, Hangzhou, Zhejiang 310012, P.R. China

E-mail: liuqin20051224@aliyun.com

Key words: microRNA, miR-146a, breast cancer, migration, RhoA patients, and thus, it is crucial to understand the molecular and cellular mechanisms that cause primary tumors to metastasize.

RhoA is a prototypical member of the Rho GTPase family, and plays an important role in the regulation of cytoskeleton reorganization. RhoA regulates signal transduction from cell surface receptors to intracellular target molecules and is involved in a variety of biological processes, including cell motility (2), morphogenesis (3), cytokinesis (4,5) and tumor progression $(6,7)$. Rho proteins bind to and activate a number of downstream effectors, leading to a cascade of biochemical responses. Previous studies have found that RhoA is upregulated in several types of human cancers, including colorectal $(8,9)$ and breast cancer $(10,11)$, hepatocellular carcinoma $(12,13)$, gastric cancer $(14,15)$, and alteration of the expression levels of intracellular RhoA can directly affect the process of invasion and metastasis in a variety of tumors $(7,16,17)$.

MicroRNAs (miRNAs) are small, endogenous, non-coding RNAs with 20-25 nucleotides that regulate the expression of hundreds of target genes. miRNAs bind target mRNA sequences through canonical base pairing between the seed sequence, which includes nucleotides 2-8 from the 5'-end, and the complementary sequence found in the 3 ' untranslated region (3'UTR) of its target mRNA (18-20). Recently, numerous studies have demonstrated that miRNAs suppress the expression of cancer-related genes and reduce tumorigenesis and metastasis in breast and several other cancers (21-23).

miR-146a was found to act as a tumor suppressor by directly regulating $\mathrm{NF}-\kappa \mathrm{B}$ expression and affecting tumor cell signaling pathways (24). miR-146a is involved in cell proliferation and influences cancer metastasis in various types of tumors, including breast cancer (25-27). However, the regulation of breast cancer metastasis by miR-146a is a complex biological network. Therefore, the regulatory mechanisms of breast cancer metastasis by miR-146a warrant further in-depth study.

In the present study, among metastasis-related genes as potential targets of miR-146a, an inverse correlation was noted between RhoA and miR-146a expression in breast cancer cell lines. Furthermore, we found that miR-146a specifically caused the downregulation of RhoA expression. miR-146a was also found to directly target the RhoA 3'UTR, thereby downregulating breast cancer cell migration and invasion. The present study aimed to elucidate the interrelation between miR-146a and RhoA, searching for promising molecular targets to inhibit 
metastasis and potential anti-metastatic agents for possible use in breast cancer therapy.

\section{Materials and methods}

Cell culture. Human breast cancer cell lines, MCF-7 and MDA-MB-231, were maintained in Dulbecco's modified Eagle's medium (DMEM) supplemented with $10 \%$ fetal bovine serum (FBS) and 1\% antibiotics (Gibco, Carlsbad, CA, USA). The cells were incubated at $37^{\circ} \mathrm{C}$ in a $5 \% \mathrm{CO}_{2}$ humidified atmosphere until reaching $75 \%$ confluency. To block the function of RhoA, the RhoA inhibitor exoenzyme C3 transferase protein (C3) (Cytoskeleton, Denver, CO, USA), was added to the culture medium at a final concentration of $2.0 \mu \mathrm{g} / \mathrm{ml}$.

Transfection. miR-146a mimics, miR-146a inhibitor, and control oligo were chemically synthesized by RiboBio (Guangzhou, Guangdong, China). All transfections were carried out using Lipofectamine 2000 reagent (Invitrogen, Carlsbad, CA, USA), according to the manufacturer's instructions. The MDA-MB-231 cells were harvested $24 \mathrm{~h}$ after transfection, and various analyses were performed. Three independent experiments were carried out in duplicate.

Plasmid constructs. The 3'UTRs of the human RhoA gene (NM_001664) were PCR amplified from human genomic DNA. The primers were digested and cloned into the pmiR-RB-REPORT ${ }^{\mathrm{TM}}$ vector (RiboBio) at the XhoI and Not I sites, and named pMIR-REPORT-RhoA-wt. The primer sequences were: h-RhoA-3UTR-forward (XhoI), 5'-CTTGAC TCGAGAACCTTGCTGCAAGCACAG-3' and h-RhoA3UTR-reverse (NotI), 5'-ATTGCGGCCGCTGCCTTTAT TCTATTAGTAG-3'. Site-directed mutagenesis with miR-146a target sites in the RhoA was carried out using a site-directed mutagenesis kit (Takara, Dalian, Liaoning, China), with pMIR-REPORT-RhoA-wt as a template, and named pMIRREPORT-RhoA-mut, respectively. The primer sequences were: h-RhoA-3UTR-mut-forward, 5'-AGCCACGTCA AGAGATGGGACCCTCAGTCACAGAG-3' and h-RhoA3UTR-mut-reverse, 5'-TCCCATCTCTTGACGTGGCTCCT CTGGGAGGGAAC-3'.

Luciferase assay. MDA-MB-231 cells were seeded into a 24-well plate $24 \mathrm{~h}$ before transfection. They were transfected with $0.4 \mu \mathrm{g}$ of the pMIR-REPORT-RhoA-wt or pMIR-REPORT-RhoA-mut, $20 \mu \mathrm{M}$ miR-146a mimics or control oligo using Lipofectamine 2000. Firefly and Renilla luciferase activities were measured consecutively using a Dual-Luciferase assay (Promega, Madison, WI, USA) according to the manufacturer's instructions. In addition, $0.02 \mu \mathrm{g}$ of the Renilla luciferase vector pRL-TK (Promega) was used for normalization. Three independent experiments were performed in triplicate.

Quantitative real-time PCR analysis ( $q R T-P C R$ ). Total RNA was prepared using TRIzol, and cDNA was generated using the PrimerScript RT reagent kit and amplified using RhoA primers with SYBR Premix Ex-Taq ${ }^{\mathrm{TM}}$ kit (all from Takara). The primer sequences for RhoA were: 5'-TTTGGAGGTGGCATAGCCTT-3' (forward) and 5'-ATGTTTAGTCAGCTGGAGAGAAGAG-3' (reverse), and the primer sequences for $\beta$-actin were: 5'-CCCTGGACTTCGAGCAAGAG-3' (forward) and 5'-AATGCCAGGGTACATGGTGG-3' (reverse). For analysis of miRNA expression by qRT-PCR, the miRcute miRNA isolation kit, miRcute miRNA First-Strand cDNA synthesis kit, miRcute miRNA qPCR detection kit and miR-146a detection primer were purchased from Tiangen Biotech (Beijing, China) to detect miR-146a and the control miRNA (U6 snRNA). Amplification and detection were performed using StepOne Plus QRT-PCR system (ABI, USA). $\beta$-actin was used to normalize the expression of RhoA, whereas U6 was used to normalize the expression of miR-146a; data were calculated based on the following equation: $R Q=2^{-\Delta \Delta C t}$.

Western blot analysis. Protein was extracted from breast cancer cell lines using RIPA buffer [150 mM NaCl, $1 \%$ NP-40, 50 mM Tris- $\mathrm{HCl}$ (pH 7.4), $1 \mathrm{mM}$ phenylmethysulfonyl fluoride, $1 \mu \mathrm{g} / \mathrm{ml}$ leupeptin, $1 \mathrm{mM}$ deoxycholic acid and $1 \mathrm{mM}$ EDTA] including a protease inhibitor cocktail and phosphatase inhibitors (Sigma, St. Louis, MO, USA), according to the manufacturer's instructions. Equal amounts of protein sample $(50 \mu \mathrm{g})$ were separated by $12 \%$ SDS-PAGE and transferred to polyvinylidene difluoride (PVDF) membranes using the Bio-Rad semi-dry transfer system. Western blotting was performed using anti- $\beta$-actin and anti-RhoA (CST, Denver, CO, USA). The signals were detected with an ECL kit (Beyotime, Nantong, Jiangsu, China) following the manufacturer's instructions.

Cell migration assay. Transwell invasion assays were performed using the MDA-MB-231 cells, which were transfected with the miR-146a mimics, miR-146a inhibitor and control oligo for $24 \mathrm{~h}$. Then, the cells were resuspended in serum-free DMEM, and placed in the top chamber with a Matrigel-coated membrane. The lower portion of the chamber contained $20 \%$ FBS as a chemoattractant. After the cells were cultured for $24 \mathrm{~h}$, we washed the chambers twice with phosphate-buffered saline (PBS), and stained the chambers using crystal violet. Five random fields were randomly selected, and images were captured from each chamber. Then, the chambers were decolorized by glacial acetic acid, and quantitative analysis was carried out using the enzyme marker at $570 \mathrm{~nm}$ wavelength. Three independent experiments were carried out in duplicate.

Wound healing assay. When cell confluency was $<90 \%$ at $24 \mathrm{~h}$ after transfection, wounds were created in the confluent cells using a 200- $\mu$ l pipette tip, and any free-floating cells and debris were removed by PBS. Medium was then added, and the culture plates were incubated at $37^{\circ} \mathrm{C}$. Wound healing within the scraped wound line was observed at a 24-h time point, and representative scrape lines for each cell line were photographed. The optical distance of the wound was measured using ImageJ software. Three independent experiments were carried out in duplicate.

Statistical analysis. All data are presented as mean \pm SD of three independent experiments. Assays for characterizing the phenotypes of the cells were analyzed by Student's t-test or 

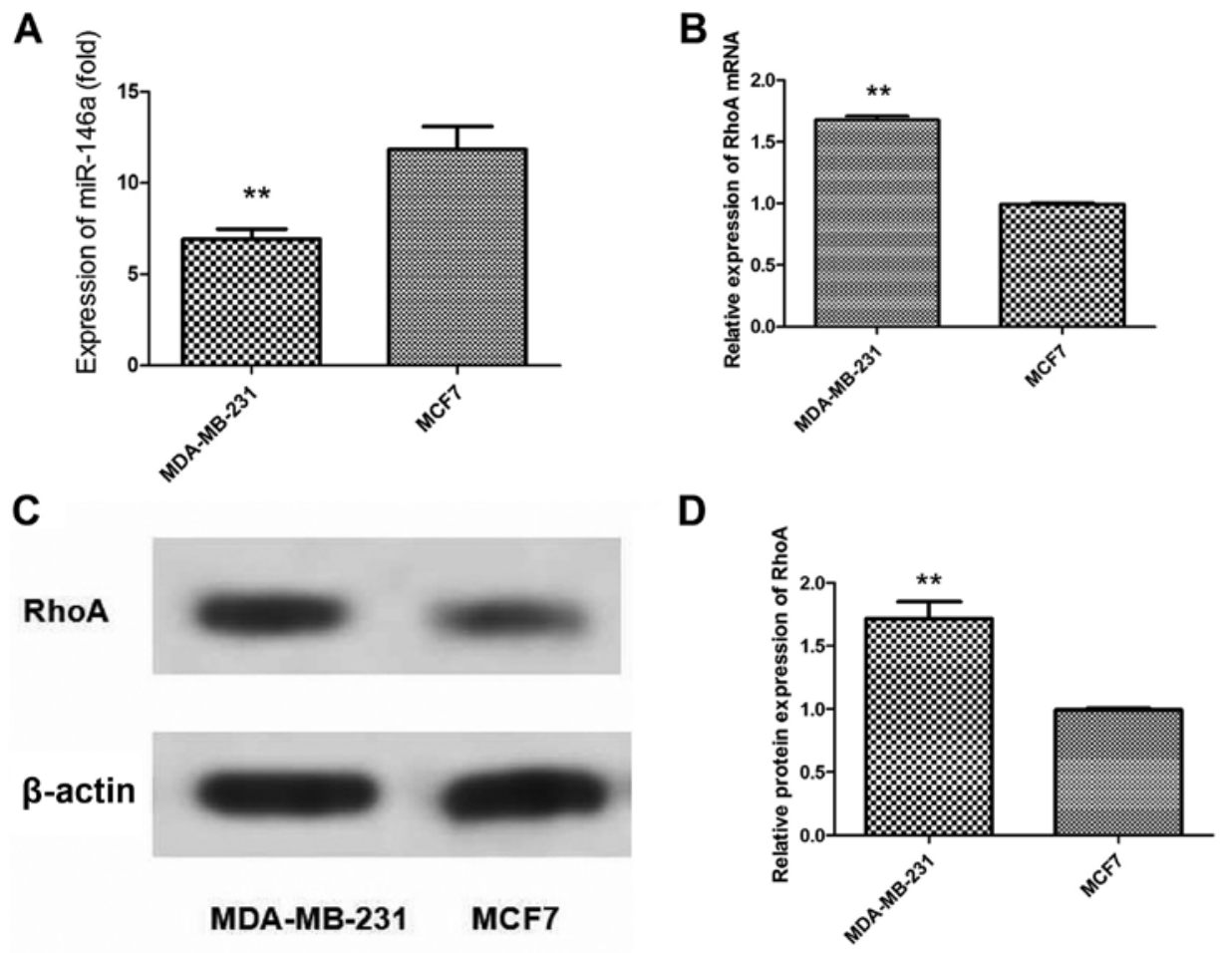

Figure 1. Differential expression of miR-146a and RhoA in breast cancer cell lines with different metastatic activity. (A) Expression of miR-146 in MDA-MB231 and MCF-7 cells was assessed by qRT-PCR. The miR-146a expression level was normalized to U6 snRNA. We used RQ $=2^{-\Delta \Delta C t}$ to represent the change in

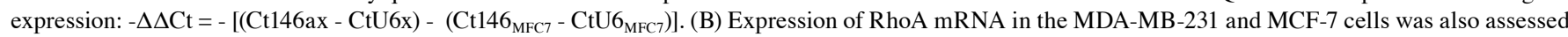
by qRT-PCR. In addition, the gene expression level was normalized to $\beta$-actin. (C and $\mathrm{D})$ The relative protein expression of RhoA was measured by western blot analysis and normalized to $\beta$-actin. The data are presented as the mean \pm SD. $n=3$; ${ }^{*} \mathrm{P}<0.05,{ }^{* * *} \mathrm{P}<0.01,{ }^{* * * *} \mathrm{P}<0.001$ vs. the MCF-7 cells.

one-way ANOVA. P-value of $<0.05$ was considered to indicate a statistically significant result. All statistical analyses were performed using the SPSS 22.0 software package (SPSS, Inc., Chicago, IL, USA).

\section{Results}

Differential expression of miR-146a and RhoA in breast cancer cells with different metastatic activity. To assess whether miR-146a levels were altered in breast cancer cell lines with different metastatic potential, two breast-derived cell lines were used to assess the relative levels of miR146a. The basal expression level of miR-146a was generally increased in the tumorigenic but weakly metastatic cell line MCF7, compared with this level in the highly metastatic cell line MDA-MB-231 (Fig. 1A). In contrast, the expression levels of RhoA mRNA and protein in MCF7 cells were obviously decreased when compared with these levels in the MDA-MB231 cells (Fig. 1B-D).

miR-146a suppresses metastatic activity in the MDA-MB-231 cells. To confirm whether change in the expression of miR146a can change the invasion and metastasis capabilities of the MDA-MB-231 cells, we upregulated miR-146a by transfecting the MDA-MB-231 cells with miR-146a mimics, and downregulated miR-146a by transfecting the MDA-MB-231 cells with the miR-146a inhibitor. The results of the Transwell experiments showed that the number of migrating cells was decreased after transfection with the miR-146a mimics. In contrast, the number of migrating cells was increased after transfection with the miR-146a inhibitor (Fig. 2A). A similar trend was observed in the wound healing assays (Fig. 2B).

Expression of RhoA varies with the amount of miR-146a expression in MDA-MB-231 cells. To confirm whether suppression of migration by miR-146a is associated with changes in RhoA expression, we transfected miR-146a mimics and the inhibitor into the MDA-MB-231 cells and detected the expression of RhoA at the mRNA and protein levels by qRT-PCR and western blotting, respectively. The qRT-PCR results showed that the expression of miR-146a was significantly altered after transfection with the miR-146a mimics and inhibitor in the MDA-MB-231 cells (Fig. 3A). In addition, the qRT-PCR results showed that the expression of RhoA mRNA was not significantly altered after transfection with the miR146a mimics and inhibitor in the MDA-MB-231 cells (Fig. 3B). However, in the western blot analysis (Fig. 3C and D), the RhoA protein concentration was decreased $(\mathrm{P}<0.01)$, when miR-146a was upregulated. In contrast, the RhoA protein concentration was increased $(\mathrm{P}<0.05)$, while miR-146a was downregulated. These results indicated that miR-146a post-transcriptionally regulated RhoA expression.

RhoA 3'UTR is a potential miR-146a target gene. To study how miR-146a may regulate migration, we proceeded to identify potential targets known to play a role in cell mobility using miRBase databases. Among the candidates surveyed, we found that the 3'UTR of the RhoA gene, which plays an important role 
A

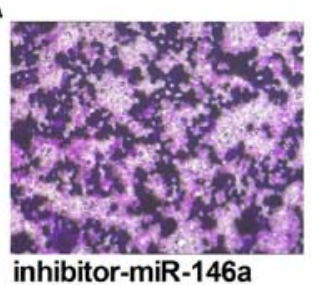

inhibitor-miR-146a

B

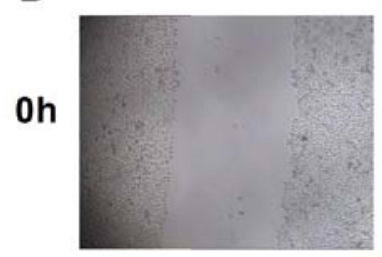

24h

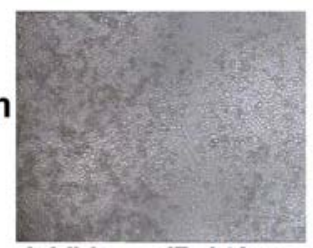

inhibitor-miR-146a

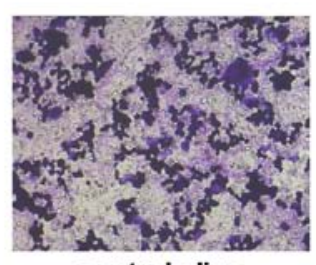

control oligo
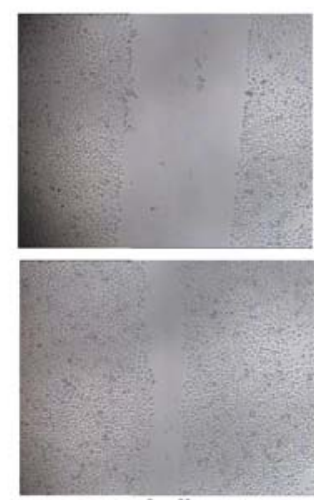

control oligo

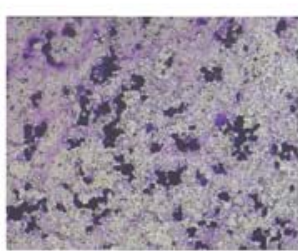

mimics-miR-146a
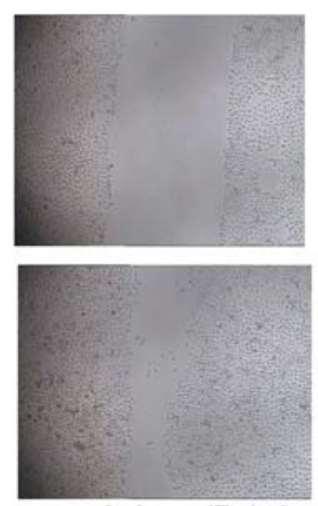

mimics-miR-146a
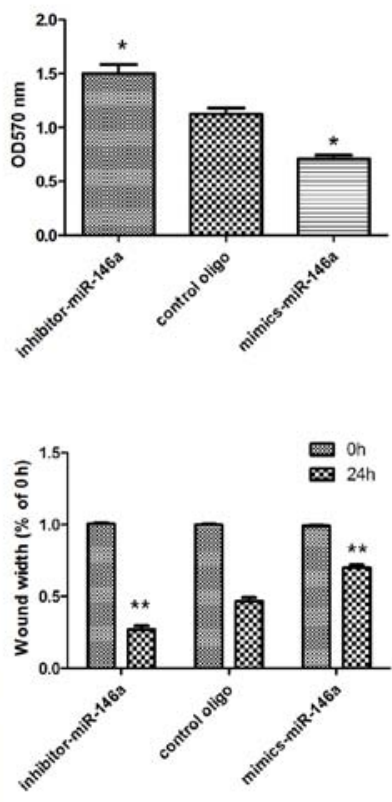

Figure 2. miR-146a suppresses the metastasis of MDA-MB-231 cells. (A) Effects of miR-146a on cell migration and invasion in the MDA-MB-231 cells was demonstrated by Transwell assay. The membrane of the upper chambers was coated with 1:7 Matrigel basement membrane matrix. Cells were harvested $24 \mathrm{~h}$ after transfection, then added (1.0x $10^{5}$ cells/well) to serum-free medium in the upper chambers, and incubated in complete medium containing $20 \%$ FBS for $24 \mathrm{~h}$. Images of cell invasion through the polycarbonate membrane stained with crystal violet (magnification, x200) are shown on the left. OD570 of crystal violet staining is shown on the right. (B) Effects of miR-146a on MDA-MB-231 cell migration by wound healing assay. MDA-MB-231 cells were plated in 6-well plates, and allowed to grow to $90 \%$ confluency in complete medium after transfection for $24 \mathrm{~h}$. Cell monolayers were wounded with a sterile micropipette tip $(200 \mu \mathrm{l})$, and cells were further incubated for $24 \mathrm{~h}$ in serum-free medium and migrated into wound surface. Images were captured at 0 and $24 \mathrm{~h}$ after wounding, and the wound widths were quantified. Data are presented as the mean $\pm \mathrm{SD} . \mathrm{n}=3 ;{ }^{*} \mathrm{P}<0.05,{ }^{* *} \mathrm{P}<0.01,{ }^{* * *} \mathrm{P}<0.001$ vs. the control group.

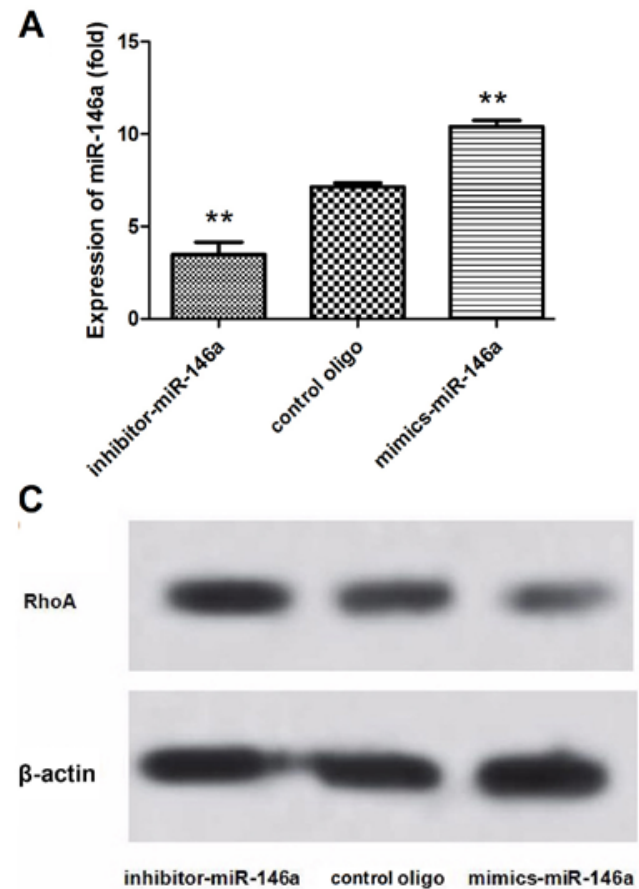

B

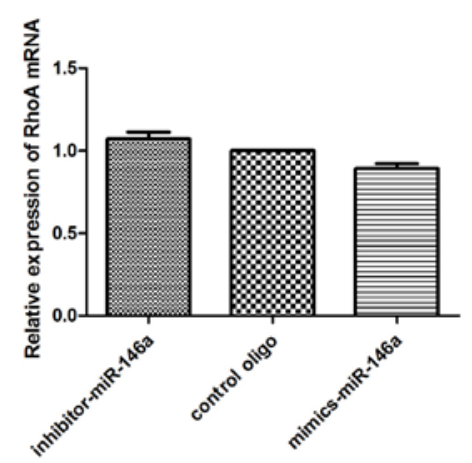

D

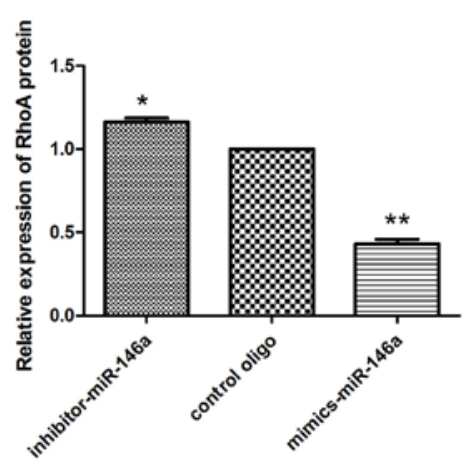

Figure 3. Expression of RhoA varies with the level of miR-146a expression in MDA-MB-231 cells. (A) To alter the amount of miR-146a expression, MDAMB-231 cells were transfected with miR-146a mimics, miR-146a inhibitor and control oligo. Gene expression levels were assessed by qRT-PCR $48 \mathrm{~h}$ after transfection. The miR-146a expression level was normalized to U6 snRNA. We used RQ $=2^{-\Delta \Delta C t}$ to represent the change in expression: $-\Delta \Delta \mathrm{Ct}=-[(\mathrm{Ct} 146 \mathrm{ax}-\mathrm{C}$ tU6x) - (Ct146 control - CtU6control)]. (B) Expression of RhoA mRNA in MDA-MB-231 cells with different levels of miR-146a expression were also measured by qRT-PCR. In addition, the gene expression level was normalized to $\beta$-actin. (C and D) The relative protein expression levels of RhoA were measured by western blot assay and normalized to $\beta$-actin. The data are presented as the mean \pm SD. $n=3$; ${ }^{*} \mathrm{P}<0.05,{ }^{* *} \mathrm{P}<0.01,{ }^{* * * *} \mathrm{P}<0.001$ vs. the control group. 
A

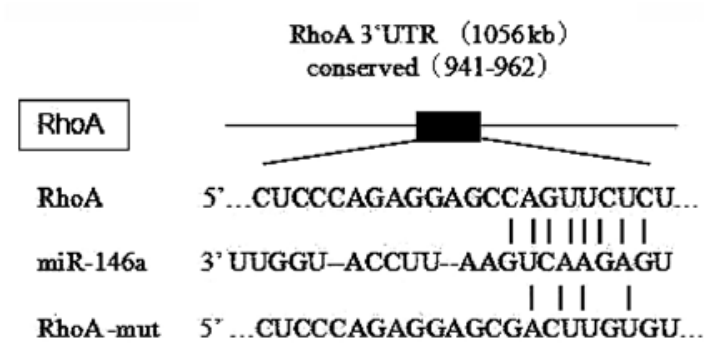

B

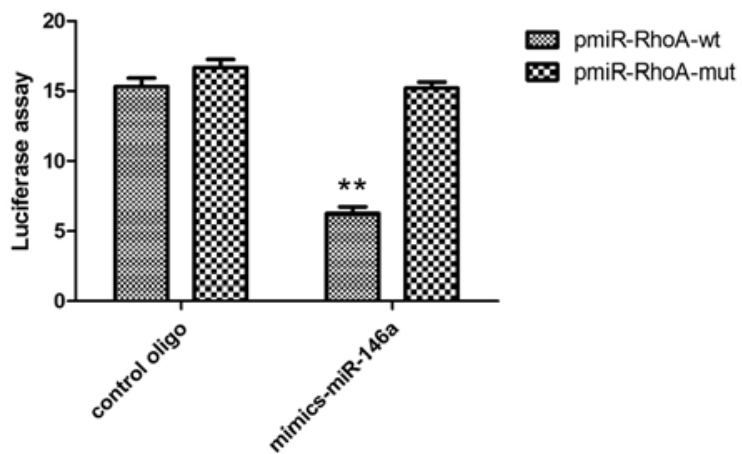

Figure 4. RhoA 3'UTR is a potential miR-146a target gene. (A) To predict and confirm that RhoA 3'UTR contains a conserved region that may serve as a binding site for miR-146a, the conserved region was determined by the miRanda algorithm. (B) A luciferase reporter of Rho A 3'UTR was constructed, which contained the binding sites of miR-146a by bioinformatics as predicted. Meanwhile the luciferase reporter of Rho A 3'UTR containing mutated binding sites of miR-146a was also constructed. miR-146a inhibits the luciferase activity of the reporter containing the 3'UTR of RhoA, when miR-146a mimics were transfected in the MDA-MB-231 cells. However, miR-146a did not inhibit the luciferase activity of the reporter containing a mutated sequence with the predicted target sites of the 3 'UTR of the RhoA gene. Data are presented as the mean $\pm \mathrm{SD}$. $\mathrm{n}=3 ;{ }^{*} \mathrm{P}<0.05,{ }^{* *} \mathrm{P}<0.01,{ }^{* * * *} \mathrm{P}<0.001$ vs. the control group.
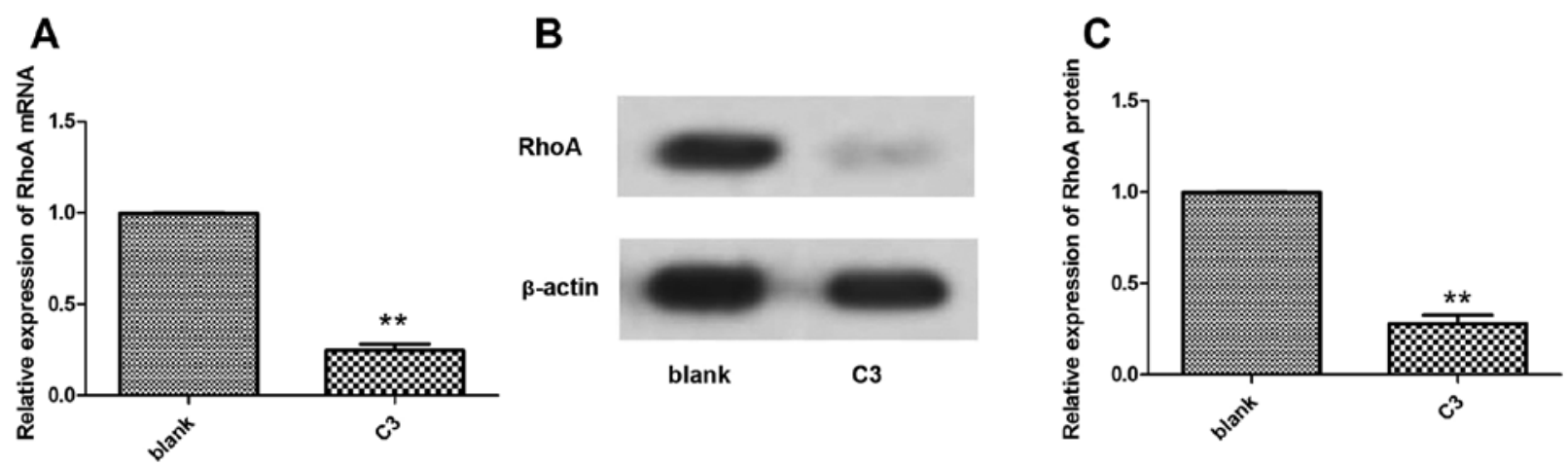

Figure 5. (A) Expression of RhoA mRNA in MDA-MB-231 cells treated with or without C3 was measured by real-time RT-PCR. In addition, the gene expression level was normalized to $\beta$-actin. (B and $\mathrm{C}$ ) The relative protein expression levels of RhoA were measured by western blot assay, and normalized to $\beta$-actin. The data are presented as the mean \pm SD. $\mathrm{n}=3 ;{ }^{*} \mathrm{P}<0.05,{ }^{* *} \mathrm{P}<0.01,{ }^{* * *} \mathrm{P}<0.001$ vs. the blank group.

in cell junction formation and stabilization $(28,29)$, contains highly conserved regions that may serve as a binding site for miR-146a as determined at microrna.org (Fig. 4A).

To further demonstrate that RhoA is a potential target of miR-146a, we generated luciferase reporters that contained the 3'UTR of the RhoA gene. To determine whether the seeding site responds to miR-146a, the reporter plasmids were introduced into the MDA-MB-231 cells along with the miR-146a mimics or the control oligo. The results showed that the reporter activity was reduced by the ectopic expression of miR-146a $(\mathrm{P}<0.001$; Fig. 4B). We also generated luciferase reporters that contained a mutated sequence within the predicted target sites of the 3'UTR of the RhoA gene to further demonstrate the interaction between miR-146a and the 3'UTR of RhoA. Data showed that the reporter activity was not reduced by the ectopic expression of miR-146a (Fig. 4B). Taken together, these results indicated that in MDA-MB-231 cells, the RhoA gene was a functional target of miR-146a.

Migratory ability exhibited no change with the levels of miR-146a expression in RhoA inhibitor C3-treated MDA-MB231 cells. To further validate whether miR-146a inhibits migration in a Rho-dependent manner, we blocked RhoA activity using the Rho inhibitor C3 at a final concentration of $2.0 \mu \mathrm{g} / \mathrm{ml}$. We found that the expression of RhoA mRNA and protein decreased after treatment with $\mathrm{C} 3$ compared with the untreated cells (Fig. 5).

Moreover, after treatment with $\mathrm{C} 3$, the migratory ability of the MDA-MB-231 cells transfected with the miR-146a mimics or the miR-146a inhibitor was neither decreased nor increased completely in the control oligo-transfected cells $(\mathrm{P}<0.05$; Fig. 6A and B). These results suggest that miR-146a does not regulate RhoA when the RhoA pathway is blocked.

\section{Discussion}

MicroRNAs (miRNAs) are a class of naturally existing small non-coding RNAs generated from large transcripts, termed pri-miRNAs and pre-miRNAs. In multicellular organisms, miRNA and the $3^{\prime}$ untranslated region (3'UTR) of its target genes are incompletely complementary, regulating the gene expression at a post-transcriptional level and affecting almost all signaling pathways, which are involved in a variety of physiological and pathological processes and also play an important regulatory role in the occurrence and progression of tumors. Research shows that miRNAs have a role in the invasion and 
A

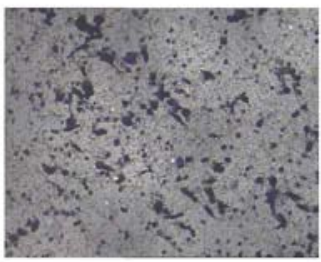

B
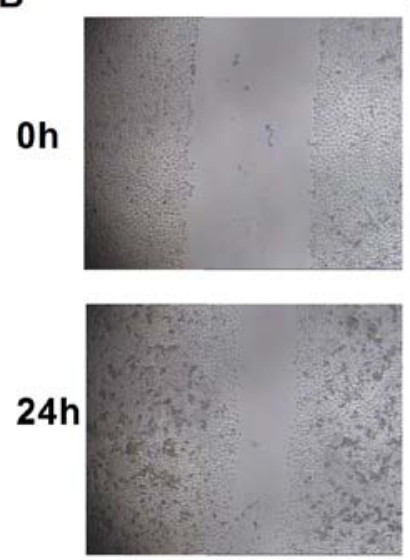

inhibitor-miR-146a
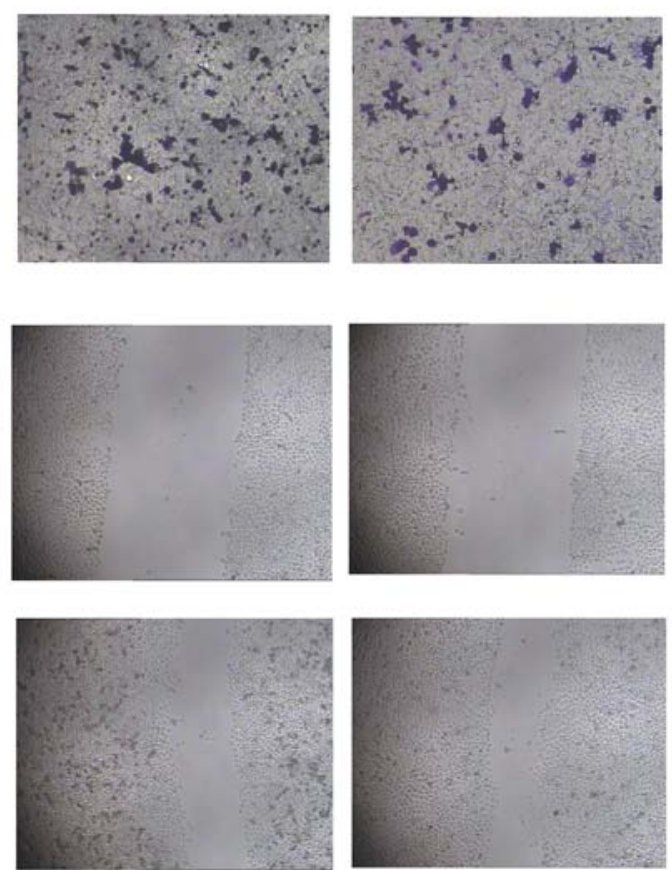

control oligo
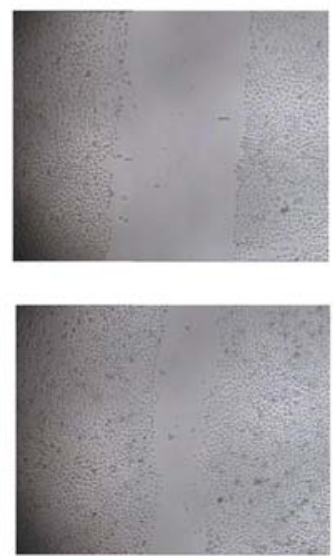

mimics-miR-146a
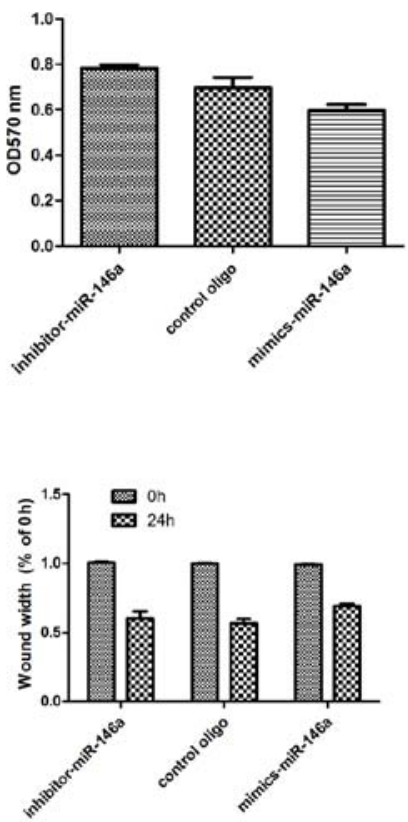

Figure 6. miR-146a suppresses the metastasis of MDA-MB-231 cells in a RhoA-dependent way. MDA-MB-231 cells were cultured in medium with $2.0 \mu \mathrm{g} / \mathrm{ml}$ Rho inhibitor C3 for $24 \mathrm{~h}$, before the expression of miR-146a was changed. (A) Effects of miR-146a on cell migration and invasion in the MDA-MB-231 cells were demonstrated by Transwell assay. Images of the cell invasion through the polycarbonate membrane stained with crystal violet (magnification, $\mathrm{x} 200$ ) are shown on the left. OD570 of crystal violet staining is shown on the right. (B) Effects of miR-146a on MDA-MB-231 cell migration by wound healing assay. Images were captured at 0 and $24 \mathrm{~h}$ after wounding and the wound widths were quantified. The data are presented as the mean $\pm \mathrm{SD} . \mathrm{n}=3 ;{ }^{*} \mathrm{P}<0.05,{ }^{* *} \mathrm{P}<0.01,{ }^{* * *} \mathrm{P}<0.001 \mathrm{vs}$. the control group.

metastasis of tumor cells (21-23). miRNAs are primarily negative gene regulators of post-transcriptional repression (30), and their targets include tumor suppressors and oncogenes (18-20).

Present studies have shown that miR-146a can inhibit the occurrence, development and metastasis in gastric and pancreatic cancer, and other tumors, while its specific mechanism remains unclear (25-27,31-33). At present, scholars have already conduct research on the regulatory mechanism of miR-146a in breast cancer metastasis. miR-146a was found to act as a tumor suppressor directly regulating $\mathrm{NF}-\kappa \mathrm{B}$ expression, and affecting the tumor cell signaling pathway (24). miR-146a is involved in cell proliferation and influences cancer metastasis in various tumor types, including breast cancer (25-27). However, the regulation of breast cancer metastasis by miR-146a is a complex biological network, and the regulatory mechanism of breast cancer metastasis by miR-146a warrants further in-depth study. The results of the present study led us to make conclusions similar to these previous studies. We confirmed that the expression of miR-146a differs in human breast cancer cell lines with different metastatic potential, and miR-146a can reduce breast cancer cell migration and invasion.

Rho/ROCK signaling channel has been researched in regares to its involvement in the molecular mechanism of tumor metastasis (34). Rho-subfamily protein is one of the members of the Ras superfamily with GTP enzyme activity, and mediates a variety of cellular effects, such as cytoskeletal reorganization, membrane trafficking, proliferation, apoptosis/ survival, cell polarity, cell adhesion, cell cycle and gene transcription (35-38). As a key member of the Rho GTPase family, RhoA has been found to be upregulated in several types of human cancers, including colorectal $(8,9)$ and breast cancer $(10,11)$, hepatocellular carcinoma $(12,13)$ and gastric cancer $(14,15)$. Previous studies have found that alteration of the expression levels of intracellular RhoA can directly affect the process of invasion and metastasis in a variety of tumors $(7,16,17,20)$. The results of our study suggest that miR-146a directly targets RhoA, and may be involved in the migration of MDA-MB-231 cells.

Based on our data, we demonstrated that in the breast cancer cell line MDA-MB-231, miR-146a directly regulates the target gene RhoA by incomplete complementarity with the 3'UTR, ultimately inhibiting the expression levels of the RhoA protein, and thereby controling the migration of cells. We used bioinformatics to determine that RhoA is closely associated with cell migration and is a target gene regulated by miR-146a. In addition, miR-146a targeting of RhoA 3'UTR was confirmed using luciferase reporter gene containing RhoA 3'UTR or specific mutated sites RhoA 3'UTR in the MDA-MB-231 cells.

The results confirmed our hypothesized that upregulated expression of miR-146a negatively inhibited the expression of RhoA protein, due to incomplete complementarity to nucleotides within the 3'UTR of RhoA. Downregulation of miR-146a expression led to increased expression of RhoA protein. However, the level of RhoA mRNA had no significant changes. The inconsistency of the degree of RhoA transcription and levels of protein expression suggest that RhoA may be subject to post-transcriptional regulation by miR-146a. According to 
the experimental results, we can infer that miR-146a may act as an anti-oncogene by suppressing MDA-MB-231 cell migration via the post-transcriptional repression of RhoA. After we blocked RhoA activity with the Rho inhibitor C3, we found that miR-146a was unable to regulate RhoA. These results further validated the possibility that miR-146a inhibited migration via a RhoA-dependent pathway.

Collectively, we demonstrated that miR-146a posttranscriptionally regulates the 3'UTR of RhoA mRNA and inhibits expression of RhoA protein, ultimately leading to suppress the migratory capacity of MDA-MB-231 cells. It is likely that miR-146a is involved in the RhoA-associated pathway, which affects the migration of the breast cancer cell line MDA-MB-231.

\section{Acknowledgements}

The present study was supported by grants from the Zhejiang Provincial Natural Science Foundation (no. LQ13H160023), the Zhejiang Provincial Natural Science Foundation (no. Y12H290042) and the Population and Family Planning Scientific Research Project of Zhejiang Province (no. 2014KYA237). We are grateful to Dr Cai Zhijian (Immunology Research Institute of Zhejiang University, Hangzhou, China) for providing the MDA-MB-231 and MCF-7 cell lines. We are grateful to Dr Xiongfa Yang (Key Laboratory of Organosilicon Chemistry and Material Technology of Education Ministry, Hangzhou Normal University, Hangzhou, China) for the statistical analysis. We also thank the members of our laboratory for the technical assistance and useful suggestions.

\section{References}

1. Jemal A, Siegel R, Ward E, Hao Y, Xu J, Murray T and Thun MJ: Cancer statistics, 2008. CA Cancer J Clin 58: 71-96, 2008.

2. Zegers MM and Friedl P: Rho GTPases in collective cell migration. Small GTPases 5: e28997, 2014.

3. Gonzalez-Billault C, Muñoz-Llancao P, Henriquez DR, Wojnacki J, Conde C and Caceres A: The role of small GTPases in neuronal morphogenesis and polarity. Cytoskeleton Hoboken 69: 464-485, 2012.

4. Chircop M: Rho GTPases as regulators of mitosis and cytokinesis in mammalian cells. Small GTPases 5: 5, 2014.

5. Jordan SN and Canman JC: Rho GTPases in animal cell cytokinesis: An occupation by the one percent. Cytoskeleton Hoboken 69: 919-930, 2012.

6. O'Connor K and Chen M: Dynamic functions of RhoA in tumor cell migration and invasion. Small GTPases 4: 141-147, 2013.

7. Orgaz JL, Herraiz C and Sanz-Moreno V: Rho GTPases modulate malignant transformation of tumor cells. Small GTPases 5: e29019, 2014.

8. Ariake K, Ohtsuka H, Motoi F, Douchi D, Oikawa M, Rikiyama T, Fukase K, Katayose Y, Egawa S and Unno M: GCF2/LRRFIP1 promotes colorectal cancer metastasis and liver invasion through integrin-dependent RhoA activation. Cancer Lett 325: 99-107, 2012.

9. Cai K, Mulatz K, Ard R, Nguyen T and Gee SH: Increased diacylglycerol kinase $\zeta$ expression in human metastatic colon cancer cells augments Rho GTPase activity and contributes to enhanced invasion. BMC Cancer 14: 208, 2014.

10. Alho I, Costa L, Bicho M and Coelho C: Low molecular weight protein tyrosine phosphatase isoforms regulate breast cancer cells migration through a RhoA dependent mechanism. PLoS One 8: e76307, 2013.

11. Dulong C, Fang YJ, Gest C, Zhou MH, Patte-Mensah C, MensahNyagan AG, Vannier JP, Lu H, Soria C, Cazin L, et al: The small GTPase RhoA regulates the expression and function of the sodium channel Nav1.5 in breast cancer cells. Int J Oncol 44: 539-547, 2014.
12. Hu T, Guo H, Wang W, Yu S, Han L, Jiang L, Ma J, Yang C, Guo Q and Nan K: Loss of p57 expression and RhoA overexpression are associated with poor survival of patients with hepatocellular carcinoma. Oncol Rep 30: 1707-1714, 2013.

13. Wang SC, Lin XL, Li J, Zhang TT, Wang HY, Shi JW, Yang S, Zhao WT, Xie RY, Wei F, et al: MicroRNA-122 triggers mesenchymal-epithelial transition and suppresses hepatocellular carcinoma cell motility and invasion by targeting RhoA. PLoS One 9: e101330, 2014

14. Duan JT, Wang XM, Zhang SQ and Zhao GJ: Effect of RhoA gene silencing on proliferation and migration of gastric MGC-803 cells. Int J Clin Exp Med 8: 14410-14415, 2015.

15. Zhou J, Hayakawa Y, Wang TC and Bass AJ: RhoA mutations identified in diffuse gastric cancer. Cancer Cell 26: 9-11, 2014.

16. Guan R, Xu X, Chen M, Hu H, Ge H, Wen S, Zhou S and Pi R: Advances in the studies of roles of Rho/Rho-kinase in diseases and the development of its inhibitors. Eur J Med Chem 70: 613-622, 2013.

17. Yu OM and Brown JH: G Protein-coupled receptor and RhoA-stimulated transcriptional responses: Links to inflammation, differentiation, and cell proliferation. Mol Pharmacol 88: 171-180, 2015.

18. De Ruyck K, Duprez F, Ferdinande L, Mbah C, Rios-Velazquez E, Hoebers F, Praet M, Deron P, Bonte K, Speel EJ, et al: A let-7 microRNA polymorphism in the KRAS 3'-UTR is prognostic in oropharyngeal cancer. Cancer Epidemiol 38: 591-598, 2014.

19. Kong W, Yang H, He L, Zhao JJ, Coppola D, Dalton WS and Cheng JQ: MicroRNA-155 is regulated by the transforming growth factor beta/Smad pathway and contributes to epithelial cell plasticity by targeting RhoA. Mol Cell Biol 28: 6773-6784, 2008.

20. Liu M, Lang N, Chen X, Tang Q, Liu S, Huang J, Zheng Y and Bi F: miR-185 targets RhoA and Cdc 42 expression and inhibits the proliferation potential of human colorectal cells. Cancer Lett 301: 151-160, 2011.

21. Liu C and Tang DG: MicroRNA regulation of cancer stem cells. Cancer Res 71: 5950-5954, 2011.

22. Nair VS, Maeda LS and Ioannidis JP: Clinical outcome prediction by microRNAs in human cancer: A systematic review. J Natl Cancer Inst 104: 528-540, 2012.

23. Tang J, Ahmad A and Sarkar FH: The role of microRNAs in breast cancer migration, invasion and metastasis. Int J Mol Sci 13: 13414-13437, 2012.

24. Bhaumik D, Scott GK, Schokrpur S, Patil CK, Campisi J and Benz CC: Expression of microRNA-146 suppresses NF-kappaB activity with reduction of metastatic potential in breast cancer cells. Oncogene 27: 5643-5647, 2008.

25. Chen G, Umelo IA, Lv S, Teugels E, Fostier K, Kronenberger P, Dewaele A, Sadones J, Geers C and De Grève J: miR-146a inhibits cell growth, cell migration and induces apoptosis in non-small cell lung cancer cells. PLoS One 8: e60317, 2013.

26. Sun Q, Zhao X, Liu X, Wang Y, Huang J, Jiang B, Chen Q and Yu J: miR-146a functions as a tumor suppressor in prostate cancer by targeting Rac1. Prostate 74: 1613-1621, 2014.

27. Zheng T, Chou J, Zhang F, Liu Y, Ni H, Li X, Zheng L, Tang T, Jin L and Xi T: CXCR4 3'UTR functions as a ceRNA in promoting metastasis, proliferation and survival of MCF-7 cells by regulating miR-146a activity. Eur J Cell Biol 94: 458-469, 2015.

28. Citi S, Spadaro D, Schneider Y, Stutz J and Pulimeno P: Regulation of small GTPases at epithelial cell-cell junctions. Mol Membr Biol 28: 427-444, 2011.

29. Wojnacki J, Quassollo G, Marzolo MP and Cáceres A: Rho GTPases at the crossroad of signaling networks in mammals: Impact of Rho-GTPases on microtubule organization and dynamics. Small GTPases 5: e28430, 2014.

30. Lovat F, Valeri N and Croce CM: MicroRNAs in the pathogenesis of cancer. Semin Oncol 38: 724-733, 2011.

31. Hou Z, Xie L, Yu L, Qian X and Liu B: MicroRNA-146a is downregulated in gastric cancer and regulates cell proliferation and apoptosis. Med Oncol 29: 886-892, 2012.

32. Li H, Xie S, Liu M, Chen Z, Liu X, Wang L, Li D and Zhou Y: The clinical significance of downregulation of mir-124-3p, mir-146a-5p, mir-155-5p and mir-335-5p in gastric cancer tumorigenesis. Int J Oncol 45: 197-208, 2014.

33. Li Y, Vandenboom TG II, Wang Z, Kong D, Ali S, Philip PA and Sarkar FH: miR-146a suppresses invasion of pancreatic cancer cells. Cancer Res 70: 1486-1495, 2010.

34. Matsuoka T and Yashiro M: Rho/ROCK signaling in motility and metastasis of gastric cancer. World J Gastroenterol 20: 13756-13766, 2014. 
35. Baranwal S and Alahari SK: Rho GTPase effector functions in tumor cell invasion and metastasis. Curr Drug Targets 12: 1194-1201, 2011.

36. Duluc L and Wojciak-Stothard B: Rho GTPases in the regulation of pulmonary vascular barrier function. Cell Tissue Res 355 675-685, 2014.
37. Ellenbroek SI and Collard JG: Rho GTPases: Functions and association with cancer. Clin Exp Metastasis 24: 657-672, 2007.

38. Street CA and Bryan BA: Rho kinase proteins - pleiotropic modulators of cell survival and apoptosis. Anticancer Res 31: 3645-3657, 2011. 